\title{
Role of Early Decompressive Craniectomy in Head Injury
}

Amit Thapa, MBBS, MS, MCh

National Institute of Neurological and Allied Sciences

Kathmandu University

Bansbari, Kathmandu, Nepal

\section{Suman Simkhada, MBBS}

National Institute of Neurological and Allied Sciences

Kathmandu University

Bansbari, Kathmandu, Nepal

Upendra P Devkota, MBBS, FRCS

National Institute of Neurological and Allied Sciences

Kathmandu University

Bansbari, Kathmandu, Nepal

\section{Address for correspondence:}

Amit Thapa, MBBS, MS, MCh

National Institute of Neurological and Allied Sciences

Kathmandu University

Bansbari, Kathmandu, Nepal

Email: dramitthapa@yahoo.com

Received, 20 September, 2011

Accepted, 15 October, 2011
$\Lambda$ s decompressive craniectomy (DC) is gaining popularity among neurosurgeons, debate is still on timing of intervention. ${ }^{13}$ Decompressive craniectomy by virtue of decreasing ICP and increasing cerebral perfusion, avoids secondary injury in head injured patients. In our practice, we find early decompressive craniectomy more beneficial than waiting till intracranial hypertension is resistant to medical measures. This not only helps salvage life but also minimizes neural loss due to raised intracranial pressure (ICP). We conducted this study to evaluate the validity of our observations.
Controversy remains as to whether early decompressive craniectomy (CDC) helps. This study was conducted to evaluate efficacy of early DC in head injury.

This retrospective study was conducted in NINAS, Kathmandu between January 2007 and October 2010. Patients admitted with head injury who underwent DC were included. DC was classified as early if performed within first 12 hours of injury and rest others as late. Early DC was performed in patients with GCS $<13$, parenchymal contusions and midline shift of $>5 \mathrm{~mm}$. Late DC was advised if intracranial pressure (ICP) was $>25 \mathrm{mmHg}$ over 15 minutes and medically intractable. Outcome was evaluated based upon Glasgow outcome score (GOS) at 6 months and duration of hospital stay.

Out of 25 patients, 12 patients had early DC and 13 late. Mean GCS at presentation was 6 and 8 respectively. Average ICP before patients were taken for late DC was 28. Mortality was 2 times higher in late DC group $(\mathrm{p}=0.158)$. Mean GOS at 6 months was 3.2 in early and 2 in late DC group $(\mathrm{p}=0.078)$. Mean hospital stay was 15.2 and 30.2 days respectively $(p=0.002)$. Subgroup analysis showed that early DC in patients with GCS $<13$ had significantly higher favorable outcome $(p=0.043)$.

Early DC in moderate to severe head injury improves outcome as well as reduces hospital stay. This being a small study, where selection criteria for early DC were not based on ICP; future study incorporating ICP criteria for selection is worthwhile.

Keywords: decompressive craniectomy, head imjury, timing, intraoranial hypertension.

\section{Materials and Methods}

We conducted a retrospective study in National institute of neurological and Allied sciences, Kathmandu. We included patients admitted over 3 years from January 2007 till October 2010. Inclusion criteria were patients with head injury with Glasgow Coma Score (GCS) at time of admission $>4$, who underwent decompressive craniectomy (DC) and whose data were retrievable and were on active follow-up. DC was considered early if done within 12 hours of injury. DC performed 12 hours of injury, were 
Thapa et al.

Table 1: Comparison of early and late decompressive craniectomy groups

\begin{tabular}{|l|l|l|l|}
\hline & $\begin{array}{c}\text { Early DC } \\
\mathrm{n}=12\end{array}$ & $\begin{array}{c}\text { Late DC } \\
\mathrm{n}=13\end{array}$ & $\mathrm{p}$ value \\
\cline { 1 - 3 } Age (range in years) & $5-52$ & $6-50$ & 0.172 \\
\cline { 1 - 3 } Age (mean in years) & 29.8 & 23.6 & \\
\hline Sex ratio (male: female) & $10: 2$ & $11: 2$ & 0.934 \\
\hline GCS at presentation (mean) & 6 & 8 & 0.425 \\
\hline $\begin{array}{r}\text { Type of DC Unilateral } \\
\text { Bilateral }\end{array}$ & 11 & 11 & \multirow{2}{*}{0.712} \\
\hline
\end{tabular}

Table 1: Comparison of early and late decompressive craniectomy groups

Table 2: Comparison of outcome in early and late decompressive craniectomy groups

\begin{tabular}{|l|l|l|l|}
\hline & $\begin{array}{l}\text { Early DC } \\
\mathrm{n}=12\end{array}$ & $\begin{array}{l}\text { Late DC } \\
\mathrm{n}=13\end{array}$ & $\mathrm{p}$ value \\
\hline Mortality & $4(33 \%)$ & $8(62 \%)$ & 0.158 \\
\hline Mean GOS at six months & 3.2 & 2 & 0.078 \\
\hline Good outcome $(\mathrm{GOS}>3)$ at six months & $6(50 \%)$ & $3(23 \%)$ & 0.161 \\
\hline Mean hospital stay in days ${ }^{\mathrm{x}}$ & 15.5 & 30.2 & 0.002 \\
\hline
\end{tabular}

${ }^{x}$ In patients who survived

Table 2: Comparison of outcome measures in early and late decompressive craniectomy groups

classified as late DC. Patients were selected for early decompressive craniectomy in presence of moderate to severe head injury with computed tomography (CT) scan showing parenchymal contusions or subdural hematoma with significant mass effect and midline shift of $>5 \mathrm{~mm}$. Late surgical decompression was advised in patients with borderline hematoma or diffuse brain injury where despite intensive medical management, intracranial pressure (ICP) remained persistently raised $(\mathrm{ICP} \geq 25 \mathrm{mmHg}$ over 15 minutes). Intensive medical management for raised ICP constituted raising head end and keeping neck straight, use of mannitol, ventilating and paralyzing patient to keep $\mathrm{PaCO}_{2}<35 \mathrm{mmHg}$ and $\mathrm{PaO}_{2}>60 \mathrm{mmHg}$, control of hyperthermia and hypotension and draining Cerebrospinal fluid (CSF) through intra-ventricular catheter (IVC). Patients who arrived after 24 hours of injury to hospital were excluded from the study.

ICP monitoring was done using intra-ventricular catheter (IVC) placed through frontal burr hole. Continuous ICP monitoring was done. Decompressive craniectomy included either unilateral frontotemporoparietal decompressive craniectomy or bi frontal decompressive craniectomy. In either case temporalis muscles were reflected inferiorly. Burr holes were made either side of sagittal sinus at posterior extent and bilaterally at keyhole and at root of zygoma. Subtemporal decompression was performed down to skull base. Dura was opened in cruciate fashion and periosteal or temporalis fascial graft was used to perform duraplasty.

Primary outcome measures were mortality and glasgow outcome score (GOS) at six months. Secondary outcome measure was duration of hospital stay in survived patients.

Statistical software SPSS for windows (version 11.5.0) was used for statistical analysis of the study. Using independent sample t test, early and late DC groups were compared for significant difference between epidemiological factors (for ordinate variables) which may affect outcome results. Similarly Pearson chi square test was used to analyze nominal variables. Outcome measures in two groups were compared similarly. $\mathrm{p}$ values less than 0.05 was considered significant.

\section{Results}

Total of 35 patients underwent DC during the study period, out of which, 25 patients met the inclusion criteria and were included in the study. Majority were males (84\%). Age of patients included in the study range from 5 years to 52 years. All patients had sustained head injury following road traffic accident. 17 patients $(68 \%)$ had severe head injury (GCS <9), $5(20 \%)$ moderate head injury (GCS 912 ) and $3(12 \%)$ minor head injury (GCS $\geq 13$ ). Twelve patients (48\%) underwent early DC and $1 \overline{3}$ late DC. The two groups were comparable in terms of age $(\mathrm{p}=0.172)$, sex $(\mathrm{p}=0.934)$, Glasgow Coma Score (GCS) at presentation (0.425) and type of decompressive craniectomy (0.712) (as shown in table 1). All patients had intracranial hematomas; however in patients who underwent ICP monitoring initially had borderline hematomas (i.e. not significant to mandate urgent surgical evacuation).

All patients who underwent late DC were on ICP monitoring and had received medical measures to decrease ICP. These patients had intra-ventricular catheters (IVC) to measure ICP. There were no ICP related complications including mal-position, hematoma formation or meningitis. Mean ICP seen in late DC group was $28 \mathrm{mmHg}$ prior to DC. Mean duration of ICP measurement prior to DC was $12+/-2$ hours. As a protocol, no patient was allowed to have elevated ICP more than $25 \mathrm{mmHg}$ for more than 15 minutes. In such cases, if CT scan revealed expanding lesion, than removal of lesion together with DC was performed, otherwise in event of brain edema; only DC was carried out.

Primary and Secondary outcome measures were compared among the groups (Table 2). There was two times higher mortality in late DC group (62\%) as compared to early DC group (31\%); however it was not statistically significant $(\mathrm{p}=0.158)$.

Patients who underwent early DC had better Glasgow outcome score at six months (Table 2 and 3), but it was not statistically significant ( $\mathrm{p}=0.161$ ). $50 \%$ patients who underwent early DC had good outcome (GOS $>3$ ) whereas only $23 \%$ in late DC group had good outcome.

After excluding all 12 deaths in the cohort, there was significant decrease in hospital stay following early DC $(p=0.02)$ in survivors, when compared to late DC. Mean hospital stay following early DC was $15.5 \pm 6$ days, whereas 
following late DC was $30.2 \pm 7.1$ days (Table 2).

Analyzing 22 patients who presented with GCS $<13$ (moderate to severe head injury), early DC shows significant improvement in GOS $(p=0.043)$. With early DC, patients had median GOS of 4 however; late GCS had median GOS of 1 .

\section{Discussion}

Kocher's observation in 1901 still holds true: "If there is no CSF pressure, but brain pressure does exist, pressure relief must be achieved by opening the skull". Decompressive craniectomy (DC) as an option to alleviate high ICP due to inoperable brain tumors was used by Harvey Cushing in $1905 .{ }^{4}$ He later used it for trauma related to war. ${ }^{5}$ Subsequently decompressive craniectomy (DC) has found to provide significant role in controlling ICP due to brain edema following trauma. Rise in ICP by brain edema, not only cause cerebral herniation (due to mechanical effect) but also leads to cerebral ischemia. Brain edema leading to refractory rise in ICP is a major prognostic factor. ${ }^{6}$ Avoiding secondary injury to brain is vital in ensuring good outcome.

Based on indications, we classify DC into 2 typesprimary or prophylactic DC which aims to avoid expected increase in ICP, and secondary DC which is performed to control ICP refractory to maximal medical management. Secondary DC is usually a part of second tier options for control of ICP. European (European Brain Injury Consortium) ${ }^{9}$, North- American (Brain Trauma Foundation) ${ }^{1}$ and international (pediatric neurosurgery) ${ }^{12}$ medical societies consider DC as last resort treatment strategy to control ICP. As DC leads to fastest control of ICP with lowest rate of complication, recent literature recommended it as first among second tier options ${ }^{6}$. Our study confirms its advantages in terms of decreasing mortality with good outcome (in patients with $\mathrm{GCS}<13$ ) and shorter hospital stay.

In this study, we included only those patients whose ICP was being monitored in late DC group; as we wanted to see effect of documented rise in ICP on timing of surgical outcome. ICP was found to be rising in almost all cases, suggesting that ICP rises earlier than radiological manifestation of raised ICP. As we excluded patients reaching hospital beyond 24 hours of injury, we literally excluded the effect of significant delay in neurosurgical care.

Even though this study used a CT based guideline for early DC ( i.e. surgically significant intracranial mass lesion), we believe that benefit of early DC can be extrapolated to patients with moderate to severe head injury who show early evidence of rising ICP or whom ICP raises with slight change in position or ETCO2. Early DC by preventing secondary damage; not only avoids loss of neuronal mass due to ischemia but also by avoiding cerebral herniation, improves overall outcome. ${ }^{2}$ However ICP monitoring in borderline cases (where cistern are open with no mass effect) is useful, as in such cases performing DC without evidence of raised ICP is not warranted. In presence of surgically significant intracranial mass lesions or compressed or absent cistern with neurological deterioration, early decompression has been shown to be beneficial. ${ }^{2,3,10}$ Polin et al found that $46 \%$ patients who underwent early surgery (within 48 hours after injury) had favorable outcome as compared to $0 \%$ after 48 hours of injury. ${ }^{11}$ Guerra et al in his prospective trial of 57 severe head injury patients, found $58 \%$ of primary DC experiencing good outcome at one year as compared to $65 \%$ of secondary DC group. ${ }^{6}$ In a prospective trial of 147 patients, younger patients ( $<50$ years) who underwent DC within 9 hours of trauma had significant better outcome. ${ }^{9}$ In pediatric group, studies have found early decompressive craniectomy (DC) useful. ${ }^{7,13,14}$ We profess DC to be performed as early as possible when indicated.

In our study cohort, early DC did not provide statistically significant benefit in survival or favorable outcome, particularly in minor head injury group. This observation can be explained by urgent action taken in presence of raised ICP (within 15 minutes), hence mitigating any detrimental effect of delay in head injured patients. Moreover in early DC group, ICP was not measure due to ethical concerns. This might have lead to some patients receive early DC when it was not required.

This study does support our protocol of undertaking ICP monitoring in all head injured cases with borderline hematoma or GCS motor $\leq 3$ or who are electively ventilated. In event of documented rise in ICP for $>15$ minutes, DC is performed along with other maneuvers to control ICP (mannitol, elective ventilation and intermittent CSF drainage).

\section{Conclusions}

Within the limitation of a non randomized trial as this one, we find early decompressive craniectomy in moderate to severe head injury improves outcome. Hospital stay is also significantly reduced in survivors. A further study based on ICP monitoring to compare effects of early versus late DC is warranted.

\section{Disclosure}

The authors report no conflict of interest concerning the materials or methods used in this study or the findings specified in this paper.

\section{References}

1. Brain Trauma Foundation, American Association of Neurological Surgeons, Joint Section on Neurotrauma 
and Critical Care: Guidelines for the management of severe head injury. J Neurotrauma 13: 641-734, 1996

2. Chibbaro S, Di Rocco F, Mirone G et al. Decompressive Craniectomy and Early Cranioplasty for the Management of Severe Head Injury: A Prospective Multicenter Study on 147 Patients. World Neurosurg 75:558-562, 2011

3. Choksey M, Crockard HA, Sandilands M. Acute traumatic intracerebral haematomas: Determinants of outcome in a retrospective series of 202 cases. $\mathbf{B r}$ J Neurosurg 7:611-22, 1993

4. Cushing H. The establishment of cerebral hernia as a decompressive measure for inaccessible brain tumor with the description of intramuscular methods of making the bone defect in temporal and occipital regions. Surg Gyn Obstet 1: 297-314, 1905

5. Cushing H. Subtemporal decompressive operations for the intracranial complications associated with bursting fractures of the skull. Ann Surg 47:641-4, 1908

6. Guerra WK, Gaab MR, Dietz H et al. Surgical decompression for traumatic brain swelling: indication and results. J Neurosurg 90:187-96, 1999

7. Josan VA, Sgouros S. Early decompressive craniectomy may be effective in the treatment of refractory intracranial hypertension after traumatic brain injury. Child's Nervous System 22:1268-1274,
2006

8. Kocher T. Die therapiedes Hirndruck und chirurgische. Eangriffe bei Hirnkrankhete. Vienna.A Holder 2626, 1901

9. Maas AI, Dearden M, Teasdale GM et al. EBICguidelines for management of severe head injury in adults. European Brain Injury consortium. Acta Neurochir 139:286-294, 1997

10. Mathiesen T, Kakarieka A, Edner G. Traumatic intracerebrallesions without extracerebral haematoma in 218 patients. Acta Neurochir (Wien) 137:155-63, 1995

11. Polin RS, Shaffrey ME, Bogaev CA. Decopressive bifrontal craniectomy in the treatment of severe refractory posttraumatic cerebral edema. Neurosurgery 41: 84-94, 1997

12. Rekate HL: Head injuries: management of primary injuries and prevention of secondary damage. Childs Nerv Syst 17:632-634, 2000

13. Ruf B, Heckmann M, Schroth I et al. Early decompressive craniectomy and duraplasty for refractory intracranial hypertension in children: results of a pilot study. Critical Care 7: R133-R138, 2003

14. Taylor A, Butt W, Rosenfeld J et al. A randomized trialof very early decompressive craniectomy in children with traumatic brain injury and sustained intracranial hypertension. Child's Nerv Syst 17:154-162, 2001 